\title{
Precise calibration of few-cycle laser pulses with atomic hydrogen
}

\author{
W C Wallace, D Kielpinski, I V Litvinyuk, and R T Sang \\ Australian Attosecond Facility, Centre for Quantum Dynamics, Griffith University, \\ Nathan, Qld, 4111, Australia
}

\begin{abstract}
Interaction of atoms and molecules with strong electric fields is a fundamental process in many fields of research, particularly in the emerging field of attosecond science. Therefore, understanding the physics underpinning those interactions is of significant interest to the scientific community. One crucial step in this understanding is accurate knowledge of the few-cycle laser field driving the process. Atomic hydrogen $(\mathrm{H})$, the simplest of all atomic species, plays a key role in benchmarking strong-field processes. Its wide-spread use as a testbed for theoretical calculations allows the comparison of approximate theoretical models against nearlyperfect numerical solutions of the three-dimensional (3D) time-dependent Schrödinger equation (TDSE). Until recently, relatively little experimental data in atomic $\mathrm{H}$ was available for comparison to these models, and was due mostly to the difficulty in the construction and use of atomic $\mathrm{H}$ sources. Here, we review our most recent experimental results from atomic $\mathrm{H}$ interaction with few-cycle laser pulses and how they have been used to calibrate important laser pulse parameters such as peak intensity and the carrier-envelope phase (CEP). Quantitative agreement between experimental data and theoretical predictions for atomic $\mathrm{H}$ has been obtained at the $10 \%$ uncertainty level, allowing for accurate laser calibration intensity at the $1 \%$ level. Using this calibration in atomic $\mathrm{H}$, both accurate $\mathrm{CEP}$ data and an intensity calibration standard have been obtained $\mathrm{Ar}, \mathrm{Kr}$, and $\mathrm{Xe}$; such gases are in common use for strong-field experiments. This calibration standard can be used by any laboratory using few-cycle pulses in the $10^{14} \mathrm{~W} / \mathrm{cm}^{2}$ intensity regime centered at $800 \mathrm{~nm}$ wavelength to accurately calibrate their peak laser intensity to within few-percent precision.

Keywords: strong-field ionization, atomic hydrogen, precision measurement, intensity calibration, carrier-envelope phase
\end{abstract}

PACS numbers: $32.80 . \mathrm{Fb}$

Submitted to: J. Phys. B: At. Mol. Opt. Phys. 


\section{Introduction}

The ionization of matter by strong laser fields drives processes that range from generation of attosecond pulses [1,2], to filamentation [3] and remote lasing [4]. The ionization dynamics are quite complex due to the highly nonlinear nature of the process with respect to variations of the laser field. It is therefore imperative that the laser field is accurately known in order to retrieve a useful interpretation of said dynamics. Atomic $\mathrm{H}$ offers unparalleled opportunities for benchmarking these dynamics; it is the simplest bound atomic system and highly precise solutions to the 3D-TDSE for the laser-matter interaction are computationally possible. By proxy, careful experiments on atomic $\mathrm{H}$ allow the retrieval of the laser parameters which drive the ionization dynamics.

In recent years, our group at the Australian Attosecond Science Facility (AASF) at Griffith University has performed strong-field measurements with atomic $\mathrm{H}[5,6,7]$ which were subsequently reviewed in [8]. We obtained quantitatively accurate data which were demonstrably free from systematic errors. The measurements from that work resulted in both the calibration of the laser peak intensity, accurate to better than $1 \%$, in addition to benchmark measurements of the carrier-envelope phase dependence of strong-field ionization (SFI) of atomic H. In this article, we review the latest work from the AASF wherein we improve upon our intensity calibration standard and CEP measurements and extend them to a secondary calibration standard via the commonly used noble gases Ar, Kr, and Xe [9, 10]. These efforts were expended in order to provide reference data on the noble gases that is known to be both accurate and precise as it is based on demonstrably accurate measurements in atomic H. The regime of laser pulse durations and peak laser intensities used in our measurements is consistent with those that are most widely used in attosecond science, providing a means for other experimental groups to accurately determine their laser parameters without the need for an atomic $\mathrm{H}$ source.

\subsection{On the importance of accuracy}

The key advantage of using atomic $\mathrm{H}$ in the laser-matter interaction is that it affords knowledge on the accuracy of the data, as opposed to precision $\ddagger$. In this Review, accuracy refers to the difference between the measured value and the true value of the quantity being measured, and precision refers to the difference between the values obtained from repeated measurements of that quantity. This distinction between accuracy and precision is highlighted in figure 1. In a well-designed experiment, ideally all measurements of a quantity should agree with one another with high precision (i.e. low statistical/random errors), however they may yet still differ from the true value. This difference is known as the systematic error of the measurement. The total uncertainty of the measurement is then derived from estimates of both the systematic and random

$\ddagger$ As given by the terminology approved by the Bureau International des Poids et Measures (BIPM), the intergovernmental body responsible for metrology and precision measurement $[11,12]$ 
errors. In the case of atomic $\mathrm{H}$, highly-accurate theoretical predictions of the true values of measurement outcomes can be calculated. If the difference between a predicted outcome and a measured outcome agree to within the uncertainty of the measurement, then the systematic error is known to be negligible and therefore it can be concluded that the measurement is known to be both precise and accurate.

(a)

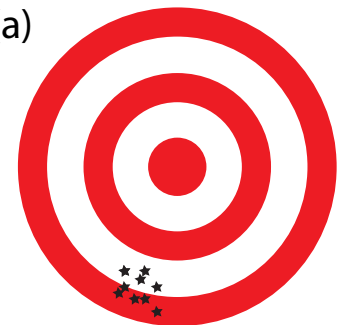

small statistical error large systematic error (b)

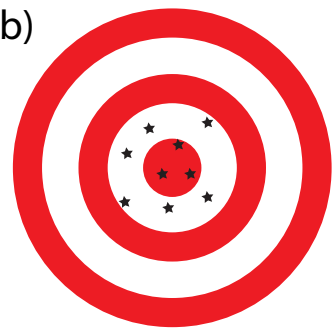

large statistical error small systematic error

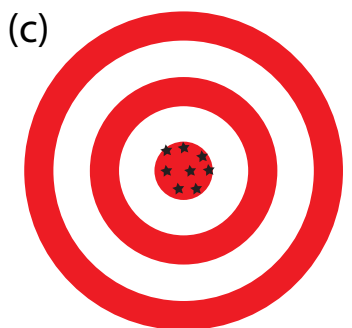

accurate precise

Figure 1: Distinction between accuracy and precision. Panel (a) shows small statistical error but large systematic error, indicative of high precision but low accuracy. Panel (b) shows large statistical error but small systematic error, indicative of low precision but high accuracy. Panel (c) shows both small statistical error and small systematic error, indicative of both high precision and high accuracy.

\subsection{Few-cycle laser pulses}

Laser pulses which have fewer than three optical pulses are defined as being few-cycle [13]. They are of particular interest due to their potential to easily access high peak powers, because rather than even distribution of the laser energy per unit time (as in continuous wave (CW) lasers), there are periods of zero laser energy between brief instances wherein the laser energy is markedly higher. The relation between average power $P_{a v g}$ and peak power $P_{p k}$ is given by (in SI units):

$$
P_{p k}=\frac{P_{a v g}}{\tau_{p} f_{r e p}}
$$

where $\tau_{p}$ is the pulse duration and $f_{r e p}$ is the repetition rate of the laser. From above, it follows that the estimated peak laser intensity, which is defined as the cycle-averaged power density, is given by:

$$
I_{p, e s t} \propto \mathrm{A}_{\mathrm{env}} \frac{2 P_{a v g}}{\pi w_{0}^{2}} \frac{1}{\tau_{p} f_{r e p}}=\mathrm{A}_{\mathrm{env}} \frac{2 P_{p k}}{\pi w_{0}^{2}},
$$

where $A_{e n v}$ is a correction factor of order unity which varies based on the temporal shape of the pulse (e.g. $A_{e n v}=0.94,0.88$, or 1 for Gaussian, sech ${ }^{2}$ or rectangular pulses respectively), $w_{0}$ is the laser beam waist, and the factor of 2 arises from the 
assumption that the spatial intensity dependence is of a Gaussian nature. Therefore it can be seen that the intensity of the laser pulse is inversely proportional to the pulse duration; few-cycle pulses facilitate access to higher laser intensities and consequently stronger fields.

Conceptually, a few-cycle pulse can be thought of as a carrier wave contained within an envelope which bounds the pulse. The mathematical description for the amplitude of the electric field oscillations of the pulse $E(t)$ can be described as [14]:

$$
E(t)=E_{0} A(t) e^{-i\left(\omega_{0} t+\phi_{0}\right)}+\text { c.c. }
$$

where $E_{0}$ is the peak amplitude of the electric field, $\omega_{0}$ is the angular frequency of the carrier wave, $\phi_{0}$ is the CEP, and $A(t)$ is an envelope function describing the temporal shape of the pulse; the full-width half-maximum (FWHM) of $\mathrm{A}(\mathrm{t})$ equals $\tau_{p}$. The peak instantaneous intensity of the pulse is then proportional to the squared value of the peak electric field according to:

$$
I(t) \propto|E(t)|^{2} \text {. }
$$

\subsection{On the importance of intensity calibration}

Photoionization of atoms and molecules from intense few-cycle laser pulses is the initial step for new techniques that measure laser CEP [15], image molecular dynamics [16], and probe electron localization [17]. Strong-field photoelectron distributions reveal complex and unexpected features such as high-energy channel-closings [18] and lowenergy structures (LES) [19]. Such features can exhibit sharp dependencies on laser peak intensity, as seen in channel-closing effects [20] and, as shown below, also for photoelectron angular distributions. Precision measurements of strong-field ionization are currently being used to explore fundamental physical processes, such as time delays in photoionization [21]. Problematically, obfuscation of these results due to small systematic offsets in the measurements have been evidenced substantially [22]. Correct experimental observation and interpretation of these processes thus necessitates both highly accurate reference data on strong-field ionization and accurate knowledge of the laser peak intensity in order for further progress to be made.

As expected for a non-perturbative process, strong-field photoionization can depend sensitively on laser intensity. For instance, channel-closing in multi-cycle laser pulses can cause order-of-magnitude changes in photoelectron spectra when the intensity changes by only a few percent [18]. Simulations also reveal significant intensity-dependent effects for few-cycle pulses. Figure 2 shows simulations of sharply intensity-dependent features of atomic $\mathrm{H}$ in the low-energy photoelectron distribution for few-cycle pulses at 800 $\mathrm{nm}$. The results are highly reliable, since they are based on solutions to the 3D-TDSE. An intensity change of only $12 \%$ causes a significant change to the sidelobes of the angular distributions. Such effects are expected to appear generically in other atomic and molecular species, and experiments with higher resolving power are necessary as they can expose physical features that are as yet still unknown. Understanding these structures requires calibration of the laser peak intensity at the few-percent level. 

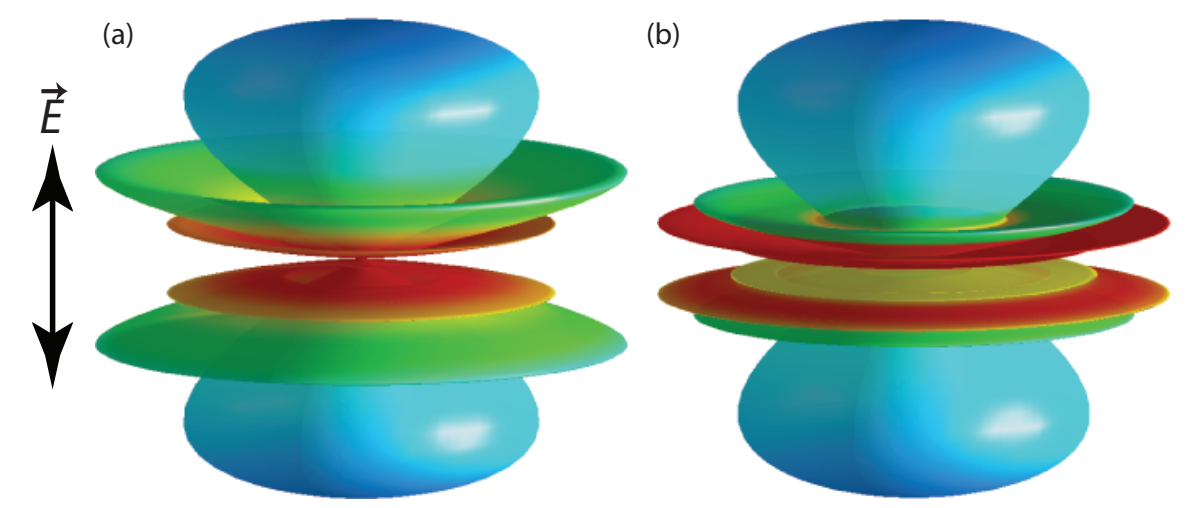

Figure 2: 3D-TDSE simulations of photoelectron angular distributions for atomic $\mathrm{H}$ (log-scale) at $2.5 \mathrm{eV}$ electron energy for linearly polarized 5.5 fs laser pulses, at laser peak intensities of (a) 1.76 and (b) $2.00 \times 10^{14} \mathrm{~W} / \mathrm{cm}^{2}$. Colour is used to guide the identification of the sidelobes and $\vec{E}$ indicates the direction of laser polarization. The simulations are averaged over the carrier-envelope phase of the laser pulse. A sharp intensity dependence is observed for an increase in laser peak intensity of only $12 \%$. From [23].

Currently, there are two in-situ methods that are considered to be the state-of-theart for calibration of the laser peak intensity. The first, proposed by Litvinyuk et al., compared theoretical simulations based upon the combination of ADK ionization rates and classical mechanics of the photoelectron and photoion momentum distributions from COLTRIMS measurements [24]. The second, by Micheau et al. [25] and Chen et al. [26] compared high-energy photoelectron spectra to the recently developed quantitative rescattering theory [27]. The accuracy of these methods is claimed to be at the $10 \%$ level, however significant approximations are inherent to the theoretical models which underpin them. To what degree these approximations affect the accuracy of the intensity calibration have not been addressed to date. The solution to this problem is to investigate a calibration method that uses photoionization of atomic $\mathrm{H}$ and subsequent comparison with highly-precise 3D-TDSE solutions in order to remove any and all doubts as to the accuracy of the method. Once this is achieved, then highlyaccurate reference data for other gas species can be obtained, allowing the creation of second-order calibration methods which are both highly accurate and transferable to laboratories without access to a source of atomic $\mathrm{H}$.

\subsection{On the importance of carrier-envelope phase}

As the pulse duration decreases, adjacent oscillations in the electric field are no longer identical. The $\phi_{0}$ term in (3) represents the position of the peak of the carrier wave $\omega_{0}$ with respect to the peak of the pulse envelope $A(t)$. A representation of this is shown in figure 3 . Changing $\phi_{0}$ varies the positions of each peak with respect to one another. For multi-cycle pulses, $\phi_{0}$ has little effect on the maximum of the electric field of the 
pulse. However, for few-cycle pulses, a change in $\phi_{0}$ from 0 (called a 'cosine' pulse) to $\pi / 2$ (called a 'sine' pulse) can have a marked effect on the maximum of the electric field. This variation in peak electric field strength is maximum when a cosine-sine variation in $\phi_{0}$ occurs.

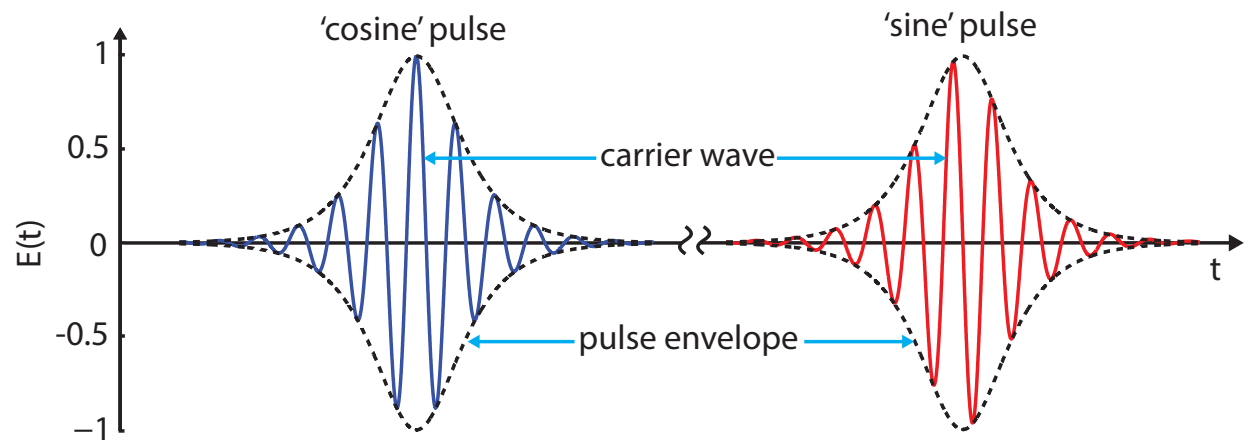

Figure 3: The carrier-envelope phase of few-cycle pulse can have a marked effect on the maximum of the electric field. As the carrier wave is shifted within the pulse envelope by a phase of $\pi / 2$ (i.e. from a 'cosine' to a 'sine' pulse), the peak electric field decreases. A cosine-sine variation in the carrier-envelope phase results in the maximum modulation of the peak electric field. From [23].

Normally, the $\phi_{0}$ term evolves rapidly from pulse to pulse, such that changes in strong-field ionization resulting from changes in peak electric field average out over a large number of pulses. However, technological advances such as the usage of frequency combs have provided the ability to stabilize $\phi_{0}$ from pulse to pulse [28]. As laser pulse durations decrease from the multi-cycle to the few-cycle regime, the maximum possible modulation in the laser peak electric field $\Delta \mathrm{E}_{\text {peak }}$ increases. An illustration of this is presented in figure 4 for $800 \mathrm{~nm}$ laser pulses, for which the period is $2.67 \mathrm{fs}$. At the pulse duration $\tau_{p}=5.5 \mathrm{fs}$, the maximum modulation of the laser peak electric field is $2.2 \%$. Controlled variation of $\phi_{0}$ is therefore a great tool for altering the laser peak electric field at the few percent level without the need to vary other laser parameters, such as pulse duration or focused spot size.

\section{Experimental apparatus}

The apparatus used for the latest AASF atomic $\mathrm{H}$ experiments consists of a few-cycle laser beam focused into a beam of atomic H. Charged particles arising from the lasermatter interaction are detected using a charged particle detection system. For brevity, a brief description of the experimental design follows; further details can be found in $[9,23,10]$ 


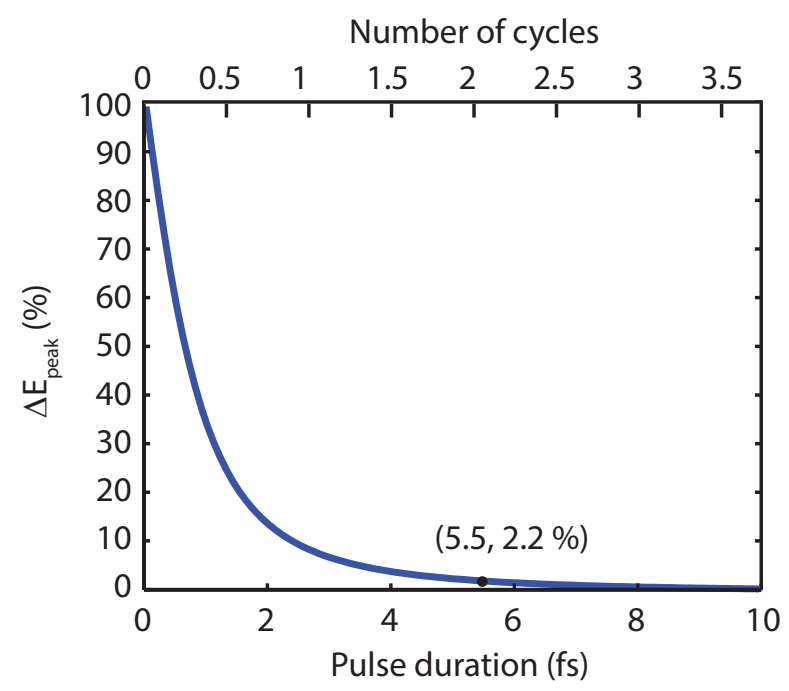

Figure 4: The percentage reduction in the peak electric field $\Delta \mathrm{E}_{\text {peak }}$ as the carrierenvelope phase of a few-cycle laser pulse is shifted by $\pi / 2$. The central wavelength $\lambda_{0}$ of the pulse is $800 \mathrm{~nm}$, corresponding to a period $T_{0}$ of $2.67 \mathrm{fs}$. For a pulse duration $\tau_{p}$ of $5.5 \mathrm{fs}$ ( $\approx 2$ cycles), the maximum modulation of the peak electric field is $2.2 \%$.

\subsection{Atomic $H$ discharge source}

The source of atomic $\mathrm{H}$ in our experiments is prepared by dissociation of molecular $\mathrm{H}_{2}$ into its constituent $\mathrm{H}$ atoms. This process is straightforward in principle, and can be achieved a number of ways: microwave discharge [29], arc discharge [30], thermal cracking [31], and radio-frequency (RF) discharge [32]. Unfortunately, the dissociation process is not particularly efficient, compounded by the fact that atomic $\mathrm{H}$ has a high recombination factor at most surfaces. Precision measurements in atomic $\mathrm{H}$ therefore require a source that is both well-characterized and relatively pure.

At the AASF we use an RF discharge source that is essentially identical to that used by the group of Kleppner [33]. The source is based on the design by Slevin [32] and consists of a water-cooled Pyrex tube which is inserted into a shielded quarter-wave helical resonator. It is schematically shown in figure $5 . \mathrm{H}_{2}$ gas flows into the tube inlet with a backing pressure of $\sim 2$ mbar. The resonator, driven by an $\mathrm{RF}$ amplifier operating at $\sim 75 \mathrm{MHz}$ at variable power $(\sim 10-17 \mathrm{~W})$, imparts energy to electrons within the tube which then impact the $\mathrm{H}_{2}$ molecules; these impacts drive the dissociation process and cause a discharge to strike. The tube is kept cool by circulating chilled water $\left(\sim 10-15^{\circ}\right.$ C) through the outer jacket of the tube. The gas that emerges at the outlet of the tube consists of up to $90 \%$ atomic $\mathrm{H}$ by number, however this efficiency is highly dependent on operational parameters such as the cooling water temperature, RF frequency, RF power, cleanliness of the tube, and even exterior influences (e.g. a noticeable difference was observed by merely touching the tube while in operation). A well-optimized source is easily visualized by a bright pink glow emanating from the tube; this pink glow originates from the Balmer- $\alpha$ emission line of atomic $\mathrm{H}$. 


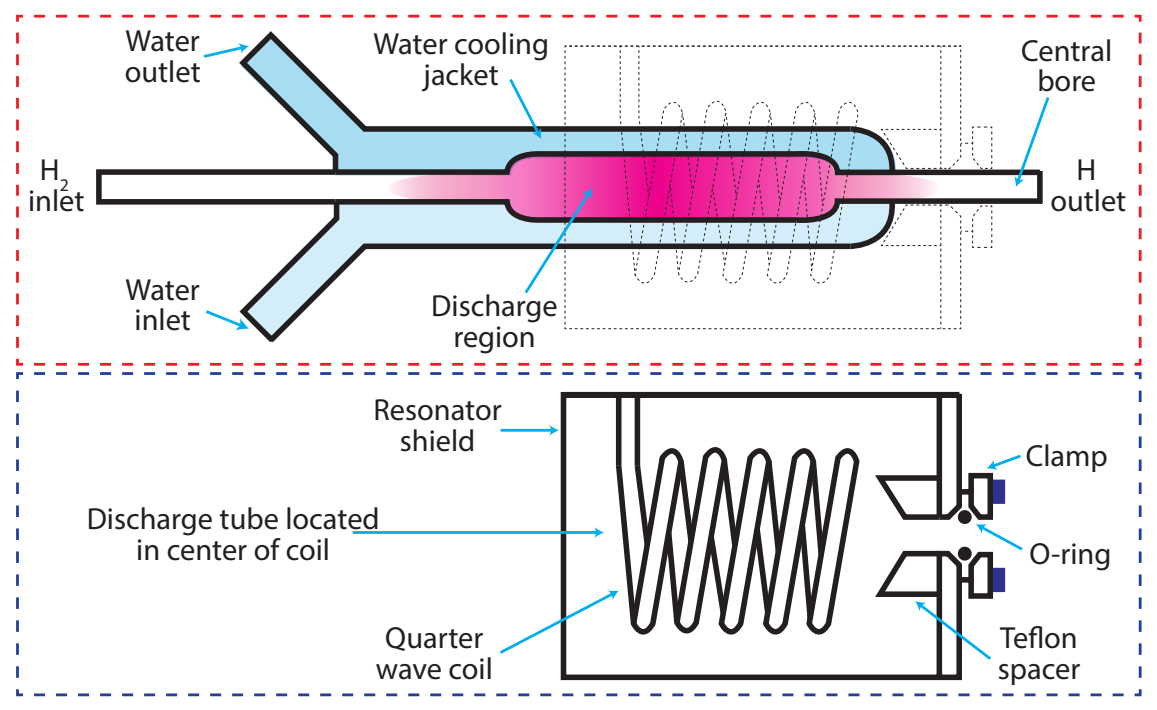

Figure 5: A schematic of the atomic $\mathrm{H}$ discharge source. $\mathrm{H}_{2}$ molecules enter the water cooled Pyrex discharge tube (red outline), are dissociated by the quarter-wave helical resonator (blue outline), and exit as atomic H. The pink glow represents the Balmer emission lines of atomic H. For clarity, the discharge tube and resonator have been separated vertically in this figure. From [23].

The atomic $\mathrm{H}$ discharge source shown in figure 5 is housed inside the source chamber which is one of three sections which comprises the atomic $\mathrm{H}$ vacuum apparatus. A schematic of the vacuum system is shown in figure 6 . The atomic beam exits the tube into the source chamber and passes through a $\sim 3 \mathrm{~mm}$ aperture into a differential pumping chamber, before passing through a second $0.5 \mathrm{~mm}$ aperture into the experiment chamber. The apertures are separated by approximately $120 \mathrm{~cm}$, resulting in a wellcollimated beam. This configuration minimizes the amount of residual $\mathrm{H}_{2}$ entering the interaction region where the laser-matter interaction occurs. However, due to the extremely low number density of the atomic $\mathrm{H}$ beam, background gases within the interaction region (predominately $\mathrm{H}_{2} \mathrm{O}$ and $\mathrm{H}_{2}$ ) still make significant contributions to the photoionization signal. These contaminants can provide as much as $9.5 \%$ of the overall signal yield.

In order to perform precision measurements of photoionization of atomic $\mathrm{H}$, contributions arising from photoionization of the background $\mathrm{H}_{2}$ and $\mathrm{H}_{2} \mathrm{O}$ must be removed. This is achieved by performing three separate photoionization measurements: (1) with the discharge turned ON, obtaining a signal $\mathrm{Y}^{\mathrm{ON}},(2)$ with the discharge turned $\mathrm{OFF}$, obtaining $\mathrm{Y}^{\mathrm{OFF}}$, and (3) with the atomic beam blocked (i.e. BCK) via a gate valve, obtaining $\mathrm{Y}^{\mathrm{BCK}}$. All other experimental parameters are made as identical as possible during these three measurements. The retrieved photoionization signal arising solely from atomic $\mathrm{H}$ is then given by:

$$
\mathrm{Y}_{\mathrm{H}^{+}}=\mathrm{Y}^{\mathrm{ON}}-\mathrm{Y}^{\mathrm{BCK}}-(1-\mu)\left(\mathrm{Y}^{\mathrm{OFF}}-\mathrm{Y}^{\mathrm{BCK}}\right),
$$




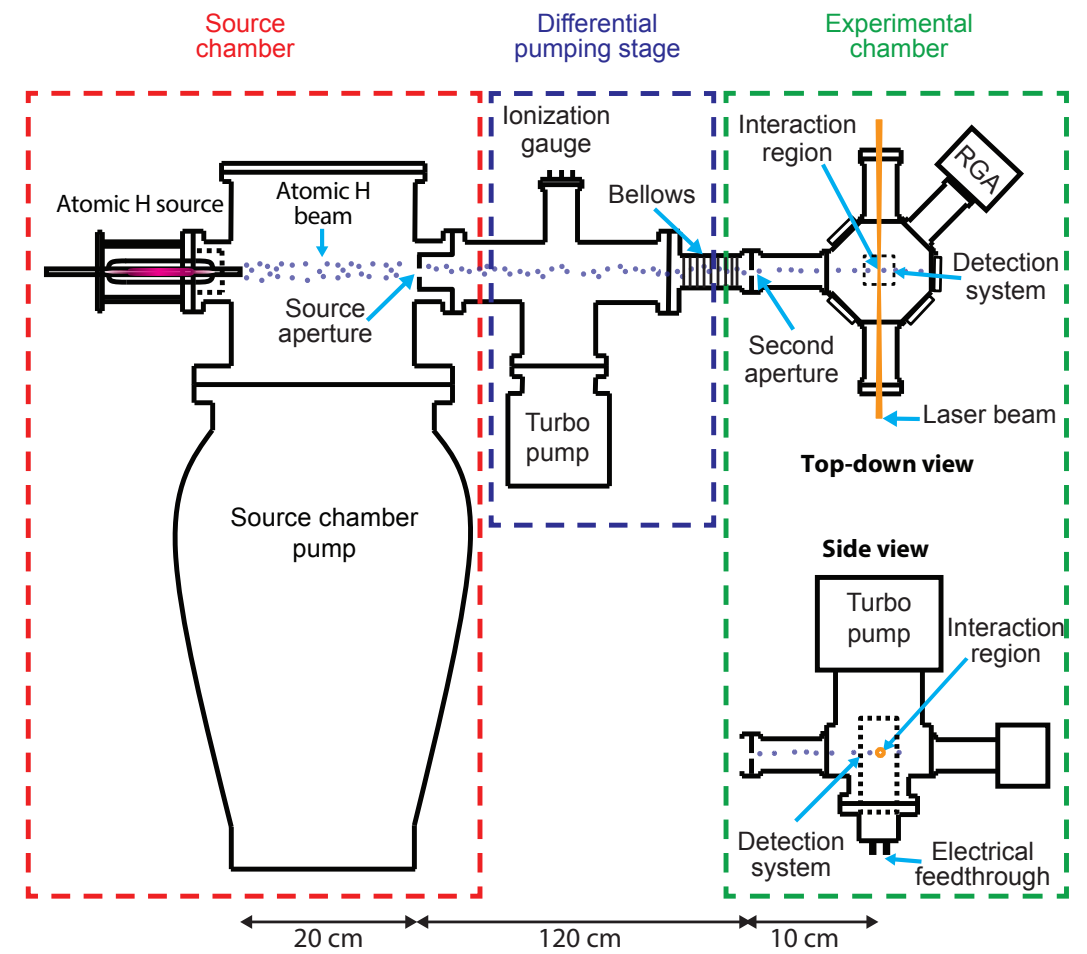

Figure 6: A schematic of the atomic $\mathrm{H}$ vacuum system. The Atomic $\mathrm{H}$ beam is created via the discharge in the source chamber (red outline), travels $20 \mathrm{~cm}$ from the exit of the discharge tube and passes through an aperture $(\sim 3 \mathrm{~mm})$ into the differential pumping stage (blue outline), travels a further $120 \mathrm{~cm}$ before passing through another aperture $(0.5 \mathrm{~mm})$ into the experimental chamber (green outline) where it intersects with the few-cycle laser beam (orange) $10 \mathrm{~cm}$ away from the last aperture. From [23].

where $\mu$ is the mass dissociation fraction which is equal to $n_{H} /\left(n_{H}+2 n_{H_{2}}\right)$, and $n_{H}$, $n_{H_{2}}$ are the number densities for $\mathrm{H}$ and $\mathrm{H}_{2}$ in their electronic ground states respectively. Accurate measurement of $\mathrm{Y}_{\mathrm{H}^{+}}$thus necessitates accurate knowledge of $\mu$. In our previous work, $\mu$ was determined using emission spectroscopy of the RF discharge $[34,35]$ to within $10 \%$ uncertainty and is detailed in [36]. For the data presented here, a new method of determining $\mu$ has been derived, but will be detailed later in this article.

\subsection{Few-cycle laser system}

The few-cycle laser system used in the AASF atomic $\mathrm{H}$ experiments is a Femtopower Compact Pro CE-Phase (Femtolasers GmbH). The laser system consists of: (I) a titanium:sapphire (Ti:S) passive Kerr-lens mode-locked, mirror dispersion-controlled oscillator which generates seed few-cycle pulses with $2.5 \mathrm{~nJ}$ energy at $80 \mathrm{MHz}$ repetition rate [37], (II) a chirped pulse Ti:S amplifier which amplifies the stretched seed pulse up to $1.1 \mathrm{~mJ}$ at $1 \mathrm{kHz}$ repetition rate [38], (III) a glass-prism compressor to re-compress the amplified pulse back down to the 30 fs duration [39], and finally (IV) a hollow-fiber compressor and dispersion compensation 'chirped' mirror set to both spectrally broaden 
and compress the pulses down to few-cycle duration [40]. The carrier-envelope phase of the laser system is stabilized using two techniques: a monolithic locking technique employed in the laser oscillator to account for 'fast' phase drifts [41], and $f-2 f$ interferometry employed after the prism compressor to negate 'slow' phase drifts [42].

The overall output of the laser system consists of $\sim 400 \mu \mathrm{J}$ pulses of $\sim 5.5$ fs duration at $\sim 800 \mathrm{~nm}$ central wavelength with a repetition rate of $1 \mathrm{kHz}$. The optical layout from section (IV) of the laser onwards is illustrated in figure 7. At the AASF, the atomic $\mathrm{H}$ apparatus is located approximately $10 \mathrm{~m}$ away from the few-cycle laser system. In order to compensate for the additional dispersion due to propagation through air, and transmission through dispersive optics, we employ additional dispersion compensating mirrors.

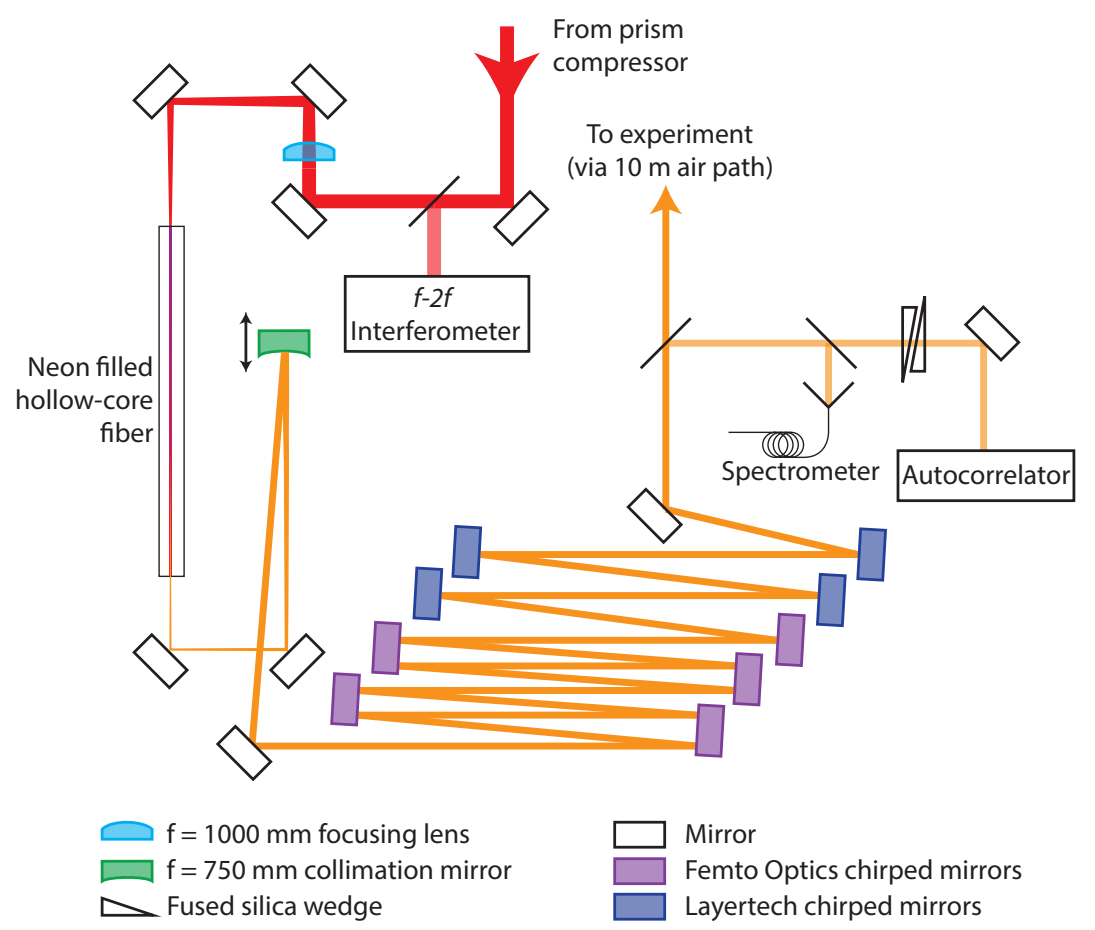

Figure 7: Neon-filled hollow-core fiber and dispersion compensating mirror set. From the prism compressor, $1.1 \mathrm{~mJ}$ sub-30 fs pulses are spectrally broadened via self-phase modulation in the fiber. The spectral broadening imparts temporal dispersion to the pulses, which is compensated for by using chirped mirror sets. A $10 \mathrm{~m}$ air-path between the laser system and $\mathrm{H}$ apparatus necessitates additional chirped mirrors to pre-compensate the positive chirp imparted via propagation through air and through dispersive optical elements. Beam diagnostics for pulse duration measurement is achieved by beamsplitter pick-off. From [23]. 


\subsection{Optical delivery}

The layout of the optics at the atomic H experimental apparatus is depicted in figure 8. Power is controlled by transmission through a set of uncoated pellicle beam-splitters on flip mounts; these pellicles impart negligible dispersion to the laser pulse owing to their $2 \mu \mathrm{m}$ thickness. Focusing of the laser through the $0.5 \mathrm{~mm}$ thick viewport window of the experimental chamber is achieved using an $\mathrm{f}=750 \mathrm{~mm}$ off-axis parabolic (OAP) mirror, which minimizes astigmatism and aberrations imparted to the beam in comparison to standard concave spherical focusing mirrors. Fine control over dispersion, and hence both the carrier-envelope phase and pulse duration, is obtained by lateral insertion of fused silica wedges (with $2^{\circ}$ wedge angle) mounted atop motorized translation stages [43]. Both a $\lambda / 2$ and $\lambda / 4$ waveplate are used to control the polarization. A residual gas analyzer (RGA) is utilized to measure partial pressures of gases within the chamber.

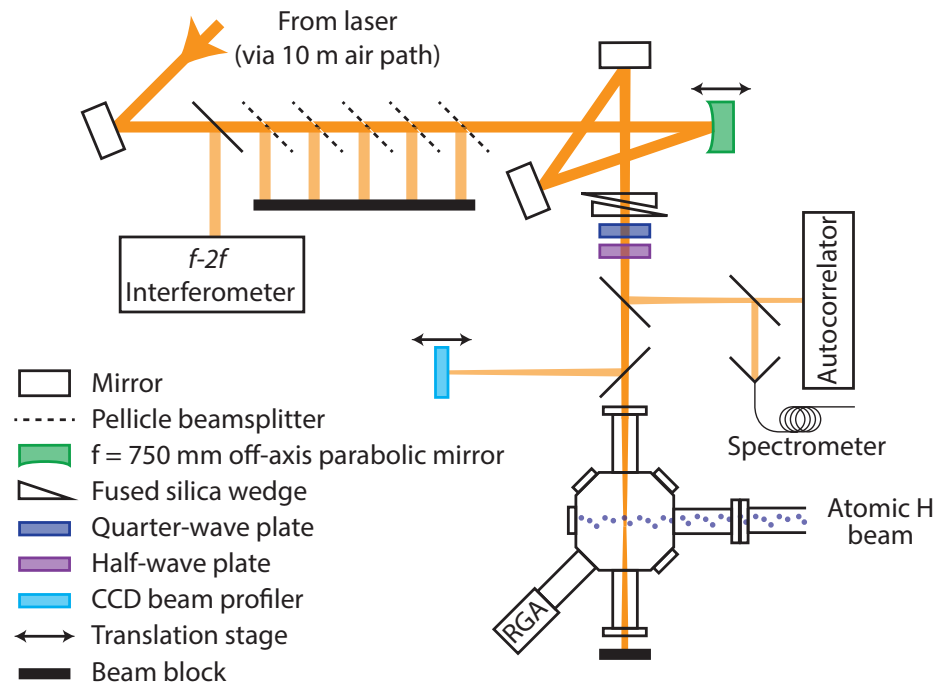

Figure 8: Optical delivery to the hydrogen apparatus. The laser beam is focused into the atomic $\mathrm{H}$ beam through a $0.5 \mathrm{~mm}$ thick viewport using a $750 \mathrm{~mm}$ off-axis parabolic mirror. Fine adjustment of the pulse duration is achieved by insertion of fused silica wedges; and control over the laser polarization is achieved by a $\lambda / 2$ and $\lambda / 4$ plate combination. Beam diagnostics are obtained via beamsplitter pick-offs located before the viewport. Power is controlled by transmission through pellicle beamsplitters, which are fixed on flip-mounts for convenient removal. From [23].

We measure beam parameters such as average laser power $P_{a v g}$, pulse duration $\tau_{p}$, and beam waist size $w_{0}$ using a thermal power meter, interferometric autocorrelation [44], and a CCD camera respectively. These diagnostics are made available via beamsplitter pick-offs on flip mounts located just prior to the viewport. From these diagnostics, and knowledge of the laser repetition rate $f_{\text {rep }}$, we are able to calculate the independently estimated laser peak intensity $I_{p, \text { est }}$ as given in equation 2 . The typical uncertainty in the calculated $I_{p, e s t}$ is on the order of $\sim 16 \%$ when accounting for 
each of the errors from the measurement diagnostics, however it is not uncommon for uncertainties in the estimated laser peak intensity to approach upwards of $50 \%$ [9].

\subsection{Charged particle detection}

The experimental data in this Review was obtained using a time-of-flight (TOF) mass spectrometer (MS) as the detection system. This system was installed as an upgrade and replacement of the channeltron detection system used in our previous work $[5,6,7]$. Briefly, TOF-MS works on the principle that similarly charged particles of differing masses born at the same spatial position within an applied electric field will acquire the same potential energy as they are accelerated through that electric field [45, 46]. For detection of positively-charged ions, the potential energy gained in the presence of the electric field is effectively converted to kinetic energy as the ion exits the electric field, with the velocity achieved by the ion being inversely proportional to its mass. In the case of negatively-charged electrons, their kinetic energy is a result of the laser-matter interaction. By allowing the particles to drift freely along some distance $d$ which is free of external electric and magnetic fields, the particles subsequently arrive at the detector at different times. The time-of-arrival of each particle allows its identification, assuming that the TOF-MS is properly calibrated.

A schematic of the TOF-MS detection system is illustrated in figure 9. The system exists entirely in vacuum, and is comprised of a top repeller plate, bottom electrode, field-free region, $70 \%$ transmissive stainless steel mesh grid, and micro-channel plate (MCP) assembly. A grounded $\mu$-metal shield encases the detection system, and reduces the effect of Earth's magnetic field by approximately two orders of magnitude in addition to alleviating any stray electric fields; this shielding facilitates the field-free drift region between the bottom electrode and the mesh grid.

The interaction region is located half-way between the repeller plate and bottom electrode, which are separated by a distance $d_{R}=10 \mathrm{~mm}$. A perspective view of the interaction region is shown in figure 10. The repeller can be either grounded or have a potential $V_{R}$ applied. The bottom electrode has a $5 \times 1.7 \mathrm{~mm}$ slit which allows free passage of charged particles. This slit is oriented at $45^{\circ}$ with respect to the propagation direction of the laser and the atomic beam and allows for easier alignment of the laser focus to both the atomic beam and the center of the MCP. The surface area of both repeller and electrode are orders of magnitude larger than the interaction volume of the laser and the atomic $\mathrm{H}$, minimizing any fringing field effects.

The laser-matter interaction produces both ions and electrons. However, ions born via the interaction with some initial kinetic energy will have a negligible velocity owing to their large mass. This increases the time taken to reach the MCP by orders of magnitude in comparison to electrons born with the same kinetic energy. We therefore facilitate ion detection by accelerating them towards the MCP by applying a positive potential $V_{R}$ at the repeller plate. For electrons born via the interaction, the initial electron velocities are already sufficient to reach the MCP in a timely fashion, negating 


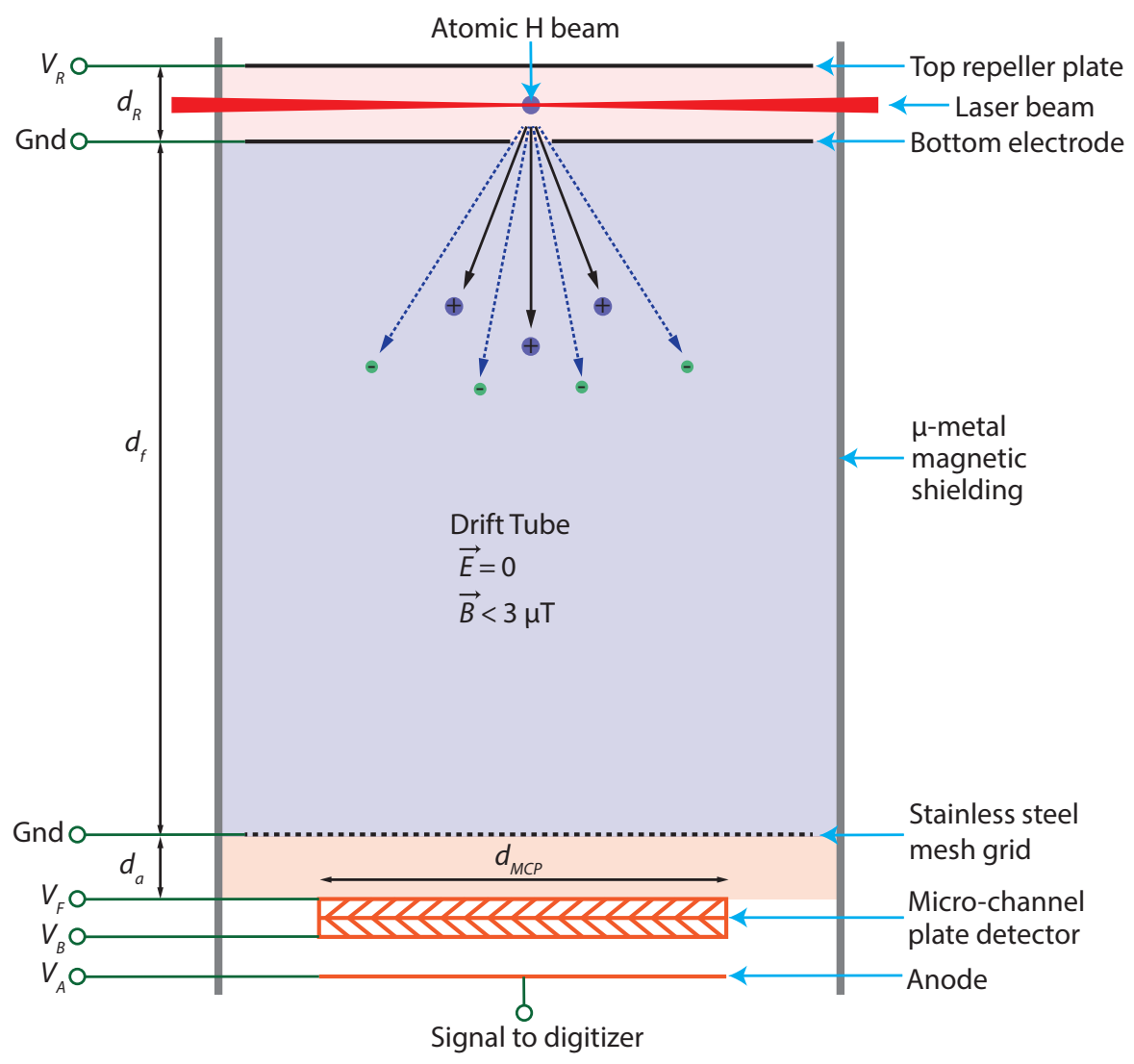

Figure 9: Time-of-flight mass spectrometer for ion and electron detection. The atomic H beam is oriented out of the page. Charged particles born via the laser-atom interaction disperse according to their mass-to-charge ratio (ions) or initial kinetic energy (electrons) in a field-free drift tube, before detection by a micro-channel plate detector. The output signal from the detector, representing the TOF of each particle, is measured as a current signal by a fast digitizer. Accelerating potentials are shown as green circles and represent applied voltages. The entire assembly is under high vacuum $\left(\times 10^{-10}\right.$ torr $)$ and that whilst depicted schematically, simultaneous electron and ion detection is not possible. From [23].

the need for an accelerating field. Hence, for electrons, $V_{R}$ is kept electrically grounded.

As the particles exit the interaction region via the slit in the bottom electrode, they are free to drift a distance $d_{f}=95 \mathrm{~mm}$ towards the MCP. Herein the particles disperse lighter ions (higher energy electrons) will traverse this distance faster than heavier ions (lower energy electrons), resulting in a shorter net time-of-flight from interaction region to MCP. For optimal detection efficiency at the MCP, the particles require an energy between 300-500 eV. This is achieved by applying a potential $V_{F}$ to the front face of the MCP, creating an electric field between the mesh grid and MCP face and accelerating the ions (electrons) when $V_{F}$ is negative (positive).

For the simplest case of a charged particle born into an electric field, the TOF can be calculated theoretically. From the seminal work of [47], an ion of mass $m_{i}$ and charge 


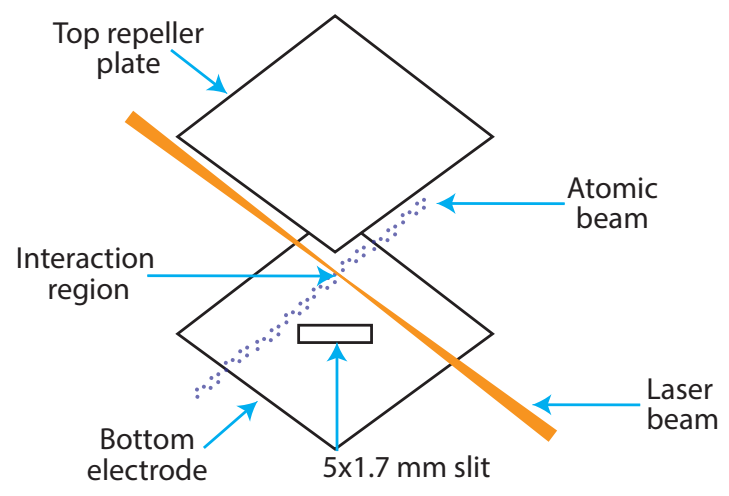

Figure 10: Perspective view of the interaction region in the time-of-flight detection system. From [23].

$q_{i}$ experiencing a single pulse of acceleration via an applied potential $V$ will traverse a field-free distance of $d$ in a time $t_{i}$ given by (in SI units):

$$
t_{i}=\frac{d}{\sqrt{2 V}} \sqrt{\frac{m_{i}}{q_{i}}}
$$

Similarly, an electron of mass $m_{e}$, charge $q_{e}$, and some initial kinetic energy $E$ will have a time of flight $t_{e}$ for the same distance $d$ given by:

$$
t_{e}=\frac{d}{\sqrt{2}} \sqrt{\frac{m_{e}}{E}}
$$

The equations for $t_{e}$ and $t_{i}$, whilst simple to start with, become more complex as additional stages are added to the TOF assembly. Nevertheless, they can still be calculated. The theoretical travel time of the charged particles through each section of the TOF-MS, denoted $d_{R}, d_{f}$ and $d_{a}$, for the accelerating potentials $V_{R}, V_{F}, V_{B}$ and $V_{A}$, is presented in table 1 . From this it is obvious that traversal times of ions for each section are proportional to the square-root of the so-called 'mass-to-charge' ratio. The total TOF is given by the sum of $t_{R}, t_{f}$, and $t_{a}$.

\subsection{Signal retrieval}

The MCP, acting as a cascaded electron multiplier, outputs a current pulse each time a charged particle strikes the front face with sufficient energy. This pulse is detected as a voltage signal, $V(t)$, which is fed to a digitizer interfaced into a computer. A LabView interface is used to view the $\mathrm{V}(\mathrm{t})$ obtained by the digitizer. An example of an ion-TOF waveform is illustrated in figure 11.

The yield of each charged particle species for a given discharge setting is obtained by integration of the baseline-subtracted $\mathrm{V}(\mathrm{t})$ over an appropriate time window which minimizes the effect of the ringing in $\mathrm{V}(\mathrm{t})$ due to a slight impedance mismatch between 
Precise calibration of few-cycle laser pulses with atomic hydrogen

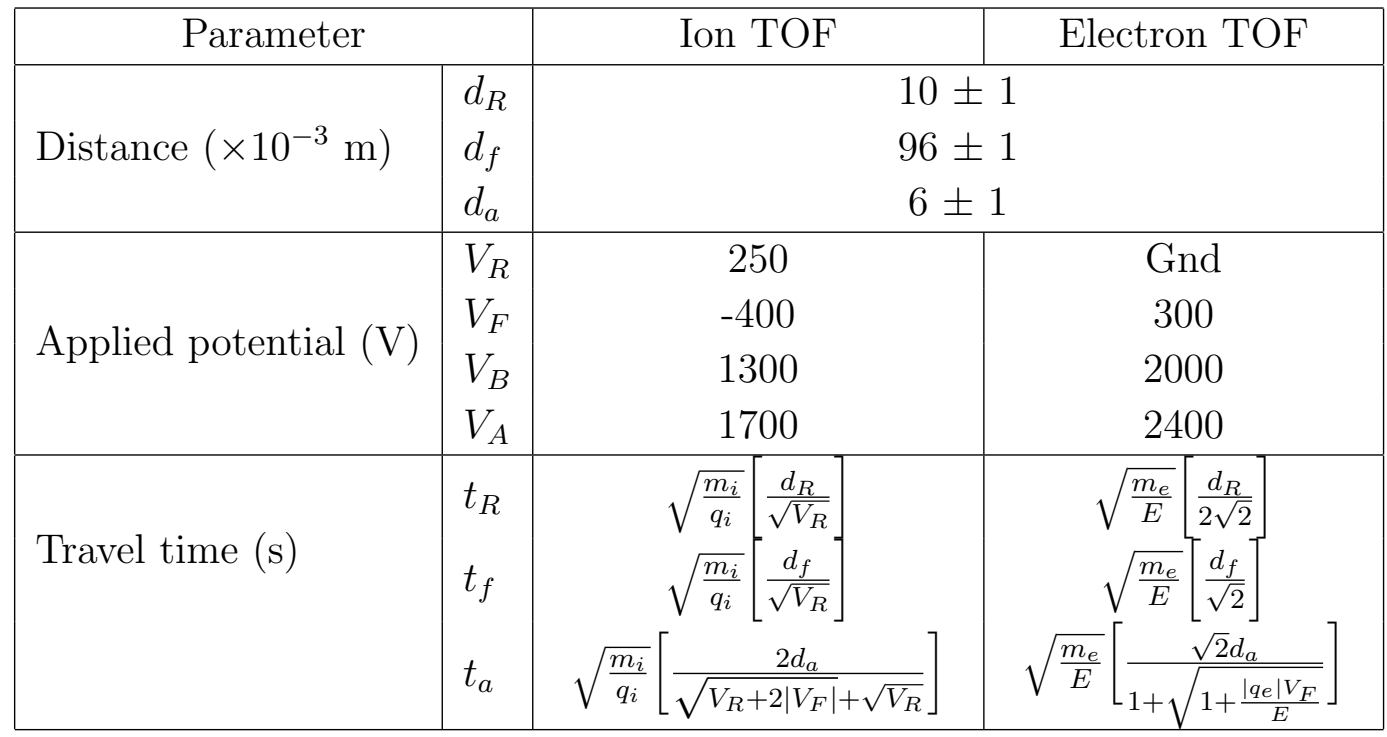

Table 1: Table of travel times for both ions and electrons (subscripts $i$ and $e$ respectively) travelling through each section of the TOF-MS for the given applied potentials. The terms $m$ and $q$ are particle masses (in $\mathrm{kg}$ ) and charges (in C) respectively, and the $E$ term is the initial kinetic energy (in $J$ ) of the ionised electron arising from the laser-atom interaction.

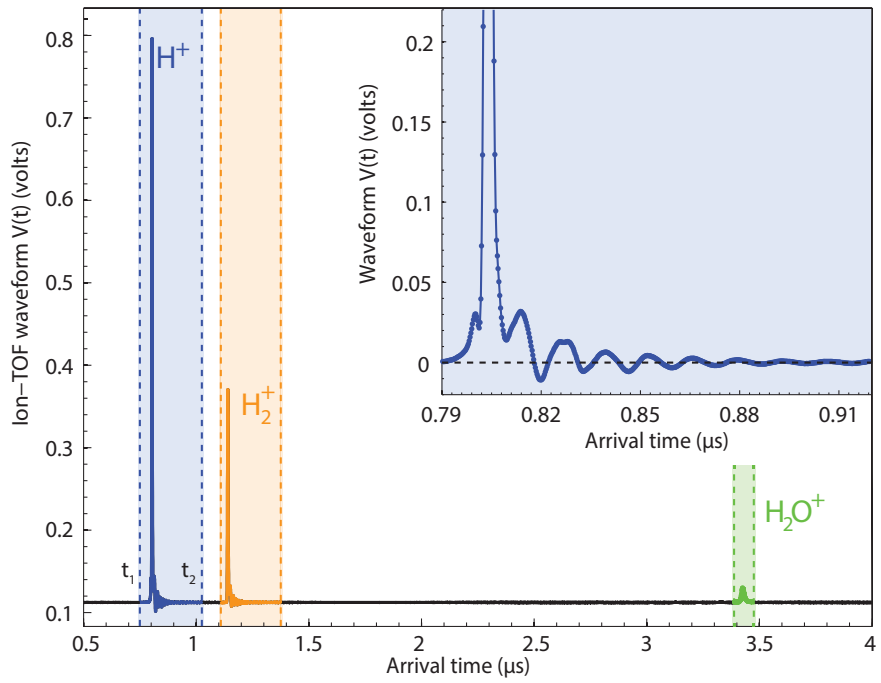

Figure 11: Main: Example voltage signal V(t) representing the ion-TOF waveform, as measured by the digitizer card. Shaded regions represent the window of integration for each ion species $\left(\mathrm{H}^{+}\right.$in blue, $\mathrm{H}_{2}^{+}$in orange, and $\mathrm{H}_{2} \mathrm{O}^{+}$in green). For example, the yield of $\mathrm{H}^{+}$ions is found by integrating $\mathrm{V}(\mathrm{t})$ between $\mathrm{t}_{1}$ and $\mathrm{t}_{2}$. Inset: The $\mathrm{H}^{+}$signal waveform after subtraction of the voltage baseline. From [23].

the MCP output and digitizer input. For example, the yield of the $\mathrm{H}^{+}$peak in figure 11 
while the discharge is $\mathrm{ON}$ is given by [23]:

$$
\mathrm{Y}_{\text {species }}^{\text {setting }}=\mathrm{Y}_{\mathrm{H}^{+}}^{\mathrm{ON}}=\int_{t_{1}}^{t_{2}} \mathrm{~V}(\mathrm{t}) \mathrm{d} t
$$

With the installation of the TOF-MS, an alternative to emission spectroscopy as a means of determining $\mu$ of the source is given as [23]:

$$
\mu=1-\frac{\mathrm{Y}_{\mathrm{H}_{2}^{+}}^{\mathrm{ON}}-\mathrm{Y}_{\mathrm{H}_{2}^{+}}^{\mathrm{BCK}}}{\mathrm{Y}_{\mathrm{H}_{2}^{+}}^{\mathrm{OFF}}-\mathrm{Y}_{\mathrm{H}_{2}^{+}}^{\mathrm{BCK}}} .
$$

Measurements of $\mu$ via the TOF-MS method agree to within uncertainty with the emission spectroscopy method.

\section{Experiments with few-cycle pulses}

Few-cycle ionization experiments with atomic $\mathrm{H}$ began in 2009 at the AASF. The rationale of the experiments was to realize the potential of atomic $H$ as the species to benchmark strong-field ionization. Our focus has been on intensity calibration and CEP effects; two aspects highly relevant to attosecond science. We have achieved quantitative agreement between theory and experiment to within a small, accurately estimated margin of error. We present out latest work here.

\subsection{Photoion measurements}

Upon installation and calibration of the TOF-MS, the new detection system was put to use by obtaining photoion yields from SFI of atomic $\mathrm{H}$ by few-cycle pulses, as opposed to the integrated photoelectron yields obtained from the old channeltron detection system as reviewed in our earlier work [8]. We measured photoion yields of atomic $\mathrm{H}, \mathrm{Y}_{\mathrm{H}}^{+}$, as a function of the independently estimated peak intensity $I_{p, e s t}$ and these

are shown in figure 12. These measurements were performed with a rapidly varying CEP over a minimum of $10^{4}$ laser shots to remove any phase effects from the few-cycle pulses. Utilizing identical error analysis techniques (predominately that of modified Allan deviation analysis [48]) and post-processing routine to those outlined in [8], we identified four sources of uncertainty in our measurements: voltage baseline subtraction, voltage waveform ringing, short-term, and long-term drifts [9].

Highly-precise 3D-TDSE solutions to the atomic H-laser interaction were provided by collaborators in the form of a differential rate $\left(I_{R}\right)$ of the ionisation probability $(P)$ with respect to both the electron energy $(E)$ and the polar angle of emission from the laser polarisation direction $(\theta)$. The ionisation probability is itself a function of $E$ and $\theta$; and of the laser peak intensity $\left(I_{0}\right)$ and laser $\operatorname{CEP}(\phi)$,

$$
I_{R}=\frac{d P\left(E, \theta, I_{0}, \phi\right)}{d \theta d E} .
$$



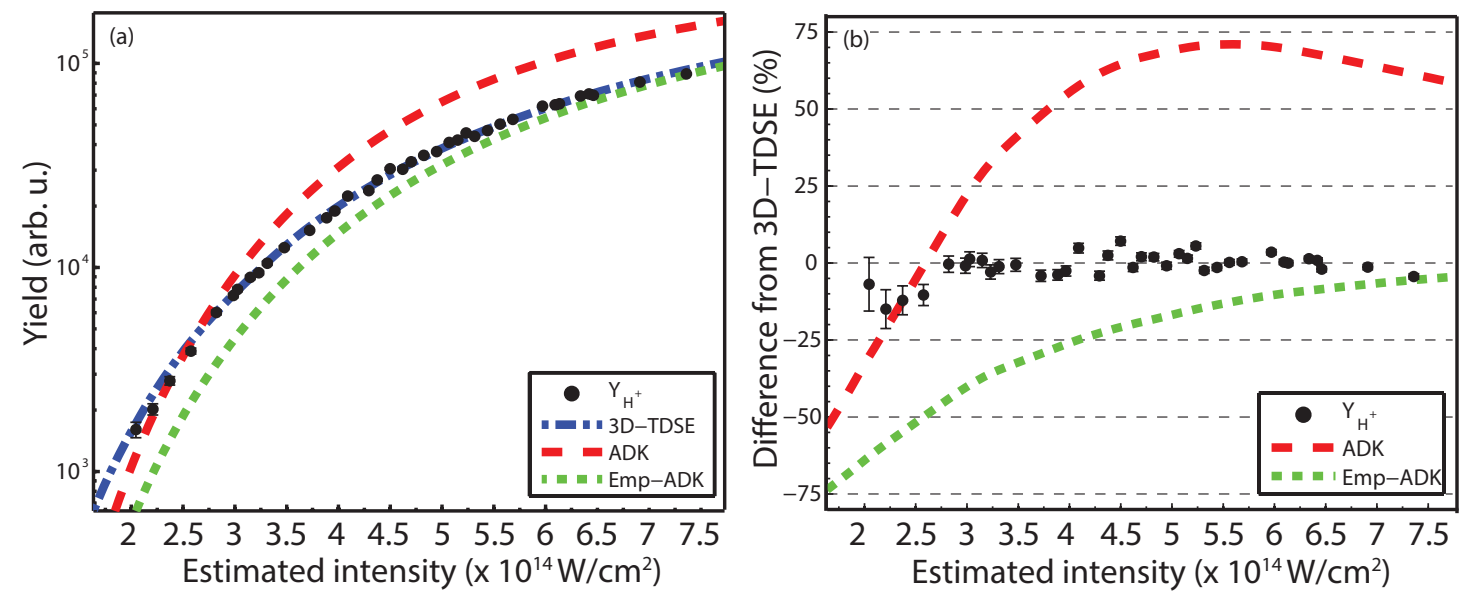

Figure 12: (a) Comparison of experimental $\mathrm{H}^{+}$yield data (circles) with theory. The excellent agreement with the 5.5 fs 3D-TDSE simulations (blue, dot-dashed line) validates the accuracy of our experimental results. As expected, standard ADK theory (ADK; red, dashed) deviates from the data at high intensities by almost a factor of two, whereas empirically-corrected ADK theory (Emp-ADK; green, dotted) exhibits marked improvement in the regime where standard ADK fails. In some cases, the error bars on the data are smaller than the symbols. (b) Percentage difference of experimental $\mathrm{H}^{+}$yield data and both ADK theories from the 3D-TDSE simulations. Copyright (2016) by the American Physical Society [9].

The resultant ionization probability $P\left(I_{0}, \phi\right)$ as a function of true peak laser intensity $I_{0}$ (as opposed to estimated peak laser intensity $I_{p, e s t}$ ) and CEP $\phi$ is given by:

$$
P\left(I_{0}, \phi\right)=\int_{0}^{\infty} \int_{0}^{2 \pi} \int_{0}^{\pi} \frac{d P\left(E, \theta, I_{0}, \phi\right)}{d E d \theta} \sin \theta d \theta d \varphi d E
$$

where $\varphi$ is the azimuthal angle and the integration is performed in spherical coordinates. Utilizing the focal-volume averaging routine outlined in [8] and noting the $4 \pi$ sr. collection angle of our TOF-MS in addition to spherical symmetry about $\varphi$, the predicted ionization signal is of the form:

$$
S\left(I_{0}\right) \propto \int_{0}^{\infty} P\left(I_{0} e^{-2 r^{2} / w_{0}^{2}}\right) r d r .
$$

As in [7], the accuracy of the acquired data set is validated by performing a comparison of the experimental $\mathrm{Y}_{\mathrm{H}^{+}}$yields to the highly-precise predictions obtained from solving the 3D-TDSE. The comparison takes the form of a least-squares fitting routine which fits the experimental data to theoretical focal-volume averaged (FVA) predictions, and is given by:

$$
\mathrm{S}_{f i t}\left(I_{p, e s t}, \kappa, \eta\right)=\kappa \cdot S\left(\eta I_{p, e s t}\right) .
$$

Here, $\kappa$ is an overall re-scaling factor of the yield which takes into account the fact that the channeltron detection efficiency and the atomic $\mathrm{H}$ beam density is unknown; and $\eta$ is an intensity scaling factor which takes into account that the precision in our estimated laser peak intensities $I_{p, e s t}$ is at best $16 \%$. 
The result of the fit in (13) is shown alongside the data in figure 12 alongside the percentage residuals. Quantitative agreement is achieved at the $10 \%$ level, with a retrieved calibration factor of $\eta=0.641 \pm 0.007$, indicating that the laser peak intensity can be accurately calibrated (i.e. absent of any systematic error) to a precision of better than $1.1 \%$. In addition to fits to 3D-TDSE we fit the data to both ADK [49] and empirically-corrected ADK [50]; these theories are commonly used as alternative theoretical approximations for SFI. Our demonstrably accurate data rules out their use as a quantitative tool for measuring the laser peak intensity.

\subsection{Transferrable calibration standard}

An additional goal of the AASF was to obtain certifiably accurate reference data on SFI of the noble gases $\mathrm{Ar}, \mathrm{Kr}$ and Xe which are commonly used as target species in laboratories using few-cycle pulses around the world. Using the same apparatus and identical conditions as the measurements in atomic $\mathrm{H}$, we obtain photoion yield data of singly-charged $\mathrm{Ar}, \mathrm{Kr}$, and Xe at laser intensities which are accurately calibrated to a precision of better than $1.1 \%$. These data are shown in figure 13, alongside fits to 3D-TDSE calculations under the single-active electron (SAE) approximation [51], ADK, and empirically-corrected ADK [50]; details found in [9].

All three theories fail to quantitatively model the photoion yield of the noble gases; this is quite unexpected and poses challenges to current theoretical models. For pure convenience, we derived a phenomenological model for each noble gas as a means of predicting the photoion signal within our regime of laser peak intensities and pulse duration. This model takes the form:

$$
S_{\text {phenom }}\left(\eta_{\mathrm{NG}} I_{p, e s t}\right)=\frac{\exp \left(-\alpha\left(\eta_{\mathrm{NG}} I_{p, e s t} / I_{c}\right)^{-1 / 2}\right)}{1+\left(\eta_{\mathrm{NG}} I_{p, e s t} / I_{c}\right)^{\gamma}} .
$$

Here, the NG subscript denotes the noble-gas species being ionized, and the parameters $\alpha, \gamma$, and $I_{c}$ are given in table 2 .

\begin{tabular}{cccc} 
Fit Parameter & $\mathrm{Ar}^{+}$ & $\mathrm{Kr}^{+}$ & $\mathrm{Xe}^{+}$ \\
\hline$\alpha$ (arb. units) & 2.84 & 4.24 & 3.71 \\
$\gamma($ arb. units $)$ & -3.03 & -2.49 & -2.69 \\
$I_{c}\left(\times 10^{14} \mathrm{~W} / \mathrm{cm}^{2}\right)$ & $3.86 \pm 0.05$ & $2.06 \pm 0.03$ & $1.18 \pm 0.03$
\end{tabular}

Table 2: Fit parameters used in Eq. (14) for the $\mathrm{Ar}^{+}, \mathrm{Kr}^{+}$, and $\mathrm{Xe}^{+}$gas targets.

Fitting the phenomenological model is performed using the same least-squares routine as earlier:

$$
S_{f i t\left(\mathrm{NG}^{+}\right)}^{(2 \mathrm{D})}\left(I_{p, e s t} ; \kappa, \eta_{\mathrm{NG}}\right)=\kappa \cdot S_{\text {phenom }}\left(\eta_{\mathrm{NG}} I_{p, e s t}\right),
$$

The result of fitting our noble-gas data to our phenomenological model is shown in figure 13 and illustrates markedly improved agreement over the other three theoretical 

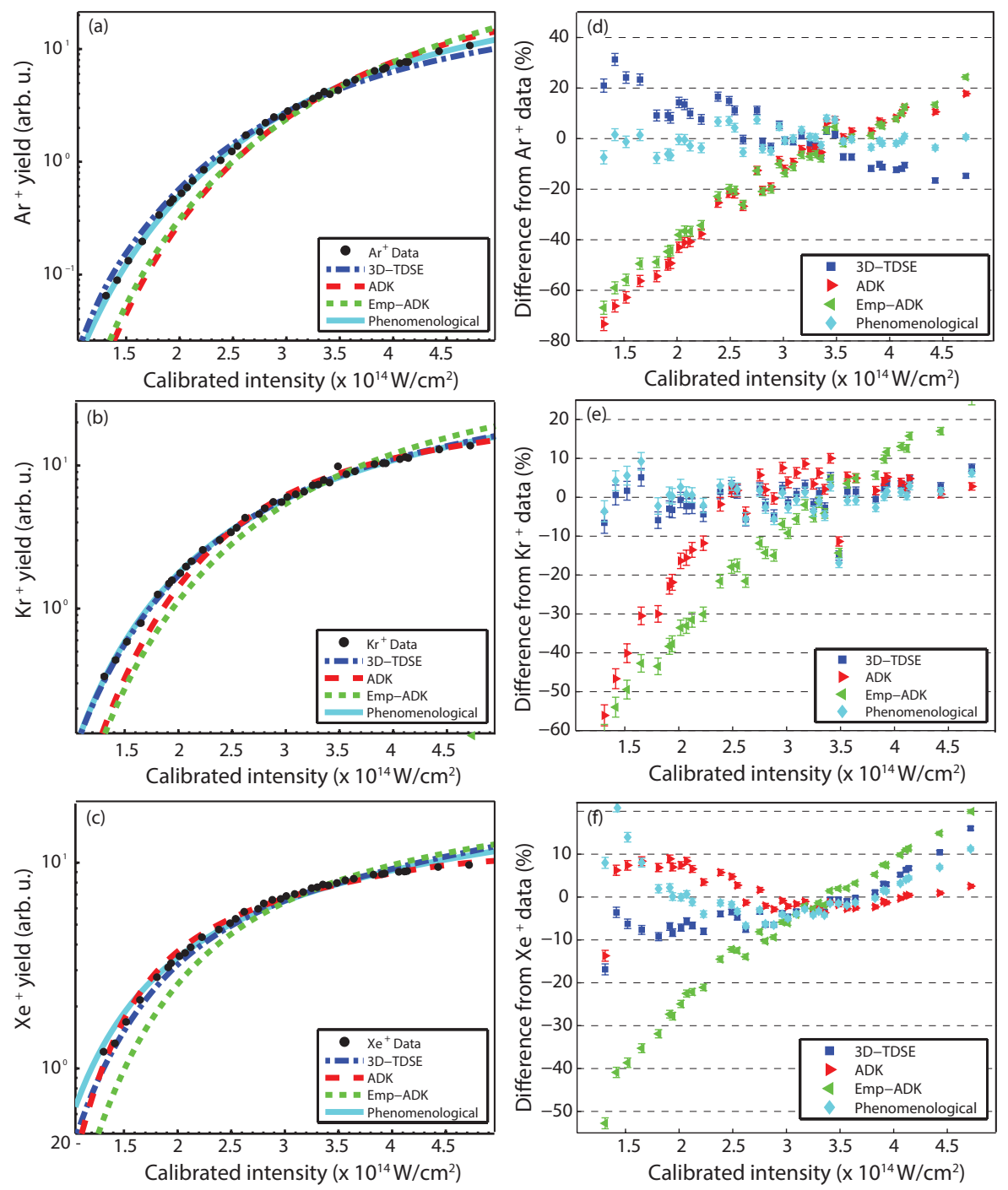

Figure 13: Left column: intensity-calibrated experimental data (black circles) for (a) $\mathrm{Ar}^{+}$, (b) $\mathrm{Kr}^{+}$, and (c) $\mathrm{Xe}^{+}$compared with theoretical predictions from 3D-TDSE (blue, dot-dashed line), ADK (red, dashed line), and Emp-ADK (green, dotted line) models, as well as the phenomenological model given by Eq. (15) (cyan, solid line). The intensity scaling parameter $\eta_{\mathrm{NG}}$ was fixed to a value of 1 , whereas $\kappa$ was allowed to vary freely in order to account for the unknown noble-gas densities. All three theories disagree with the data at the level of tens of percent. Right column: normalized residuals for (d) $\mathrm{Ar}^{+}$, (e) $\mathrm{Kr}^{+}$, and (f) $\mathrm{Xe}^{+}$, for each theoretical prediction, shown as percentage deviations from the experimental yield data for the respective gas targets. Copyright (2016) by the American Physical Society [9].

predictions. Our transferable calibration standard using our phenomenological model can be used by other few-cycle laboratories to accurately calibrate the peak laser intensity of their few-cycle lasers to within few-percent precision without the need for an atomic $\mathrm{H}$ source. 


\subsection{Carrier-envelope phase measurements}

The upgraded detection system at the AASF facilitates an improvement in the quality of CEP measurements over our previous measurements [6]. Our CEP measurements [10], are performed by motorized lateral insertion of the fused silica wedges at the hydrogen apparatus in order to vary the laser CEP $\phi$ over a range exceeding $2 \pi$. A photoelectron spectrum is obtained over $9 \times 10^{4}$ laser pulses at each wedge position, for each setting of the discharge, at calibrated peak laser intensities of 1.2 and $2.5 \times 10^{14} \mathrm{~W} / \mathrm{cm}^{2}$. Examples of energy-resolved, CEP-averaged $(\bar{\phi})$ photoelectron spectra from various atomic species are shown in figure 14 .

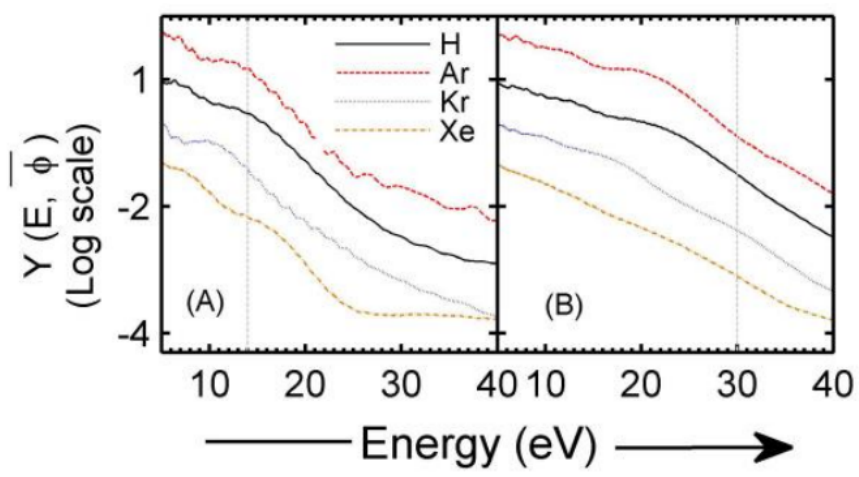

Figure 14: Photoelectron spectra averaged over CEP for atomic species atomic $\mathrm{H}$ (black), Ar (red), Kr (blue), and Xe (orange) at (A) 1.2 and (B) $2.5 \times 10^{14} \mathrm{~W} / \mathrm{cm}^{2}$. Spectra for atomic species are displaced vertically for the sake of clarity. Copyright (2017) by the American Physical Society [10].

We define the CEP-dependent photoelectron spectrum as $Y(E, \phi)$, where $E$ is the photoelectron kinetic energy. As seen in figure 14, $Y(E, \phi)$ varies over many orders of magnitude; we therefore parameterize the CEP effects by the normalized quantity $S(E, \phi)$ :

$$
S(E, \phi)=\frac{Y(E, \phi)-Y(E, \bar{\phi})}{Y(E, \bar{\phi})} .
$$

By similarly parameterizing the focal-volume averaged 3D-TDSE predictions and comparing them to our measurements, we accurately calibrate our laser CEP. An example of this comparison is shown in figure 15 wherein marked agreement between experiment and theory is achieved for the 2D 'CEP maps' which illustrate the yield dependence as a function of both CEP $\phi$ and electron energy $E$. For ease of viewing, both experiment and theory are smoothed with respect to the $E$ variable using a Gaussian filter with FWHM of $1.5 \mathrm{eV}$, and the results of $0<\phi<2 \pi$ are replicated to the range $2 \pi<\phi<4 \pi$. For convenience, the energy scale is also shown in units of ponderomotive potential $\mathrm{U}_{p}$; below $2 \mathrm{U}_{p}$ photoelectrons predominately originate from 'direct' ionization whereas above $2 \mathrm{U}_{p}$ photoelectrons originate from back-rescattering of the parent ion [52]. For back-scattering photoelectrons, CEP effects are found to be more stable. 
As the data obtained for the noble gases is taken in the same apparatus, under identical conditions as atomic $\mathrm{H}$, we therefore are able to assign an absolute CEP to the data which is free of systematic errors. Our calibrated reference data on the phase effects in noble gases only show qualitative agreement with the 3D-TDSE calculations performed under SAE, as was similar to the photoion yield data presented in [9]. Recent similar studies by Sayler et. al confirm our observations [53].

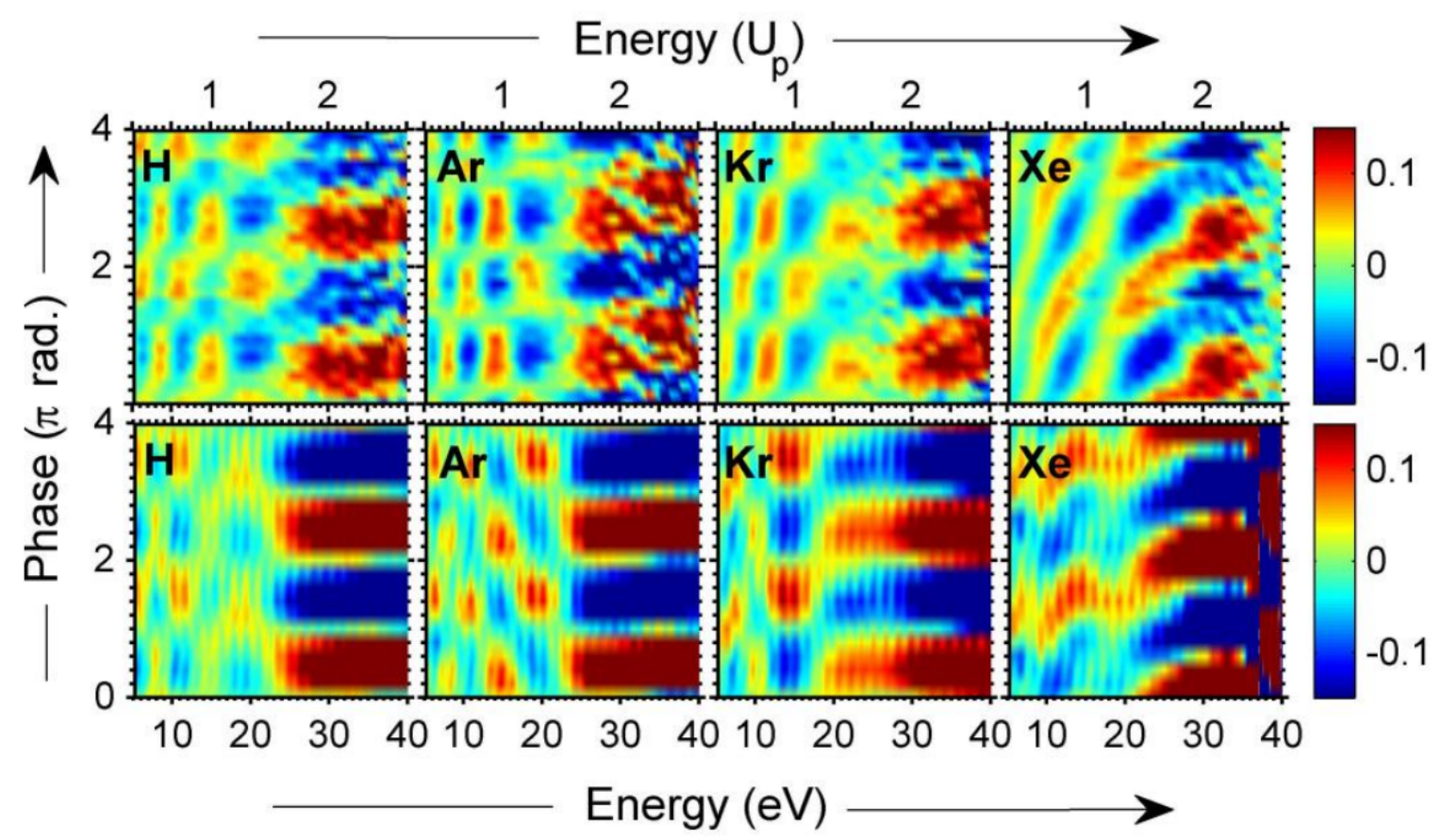

Figure 15: CEP maps for atomic species at $2.5 \times 10^{14} \mathrm{~W} / \mathrm{cm}^{2}$, based on equation (16). Top row: Experimental data. Bottom row: Theoretical simulations; exact 3D-TDSE used for atomic $\mathrm{H}$ otherwise 3D-TDSE under SAE used for noble gases. Copyright (2017) by the American Physical Society [10].

One important ramification of the reference CEP data provided herein is that calibration of the laser CEP has long been achieved using Xe as the target gas [54]. However the question remains about systematic CEP offsets since the accuracy of the 3D-TDSE under SAE models for these target species is not well characterized, as demonstrated here. By directly referencing our noble-gas data to atomic $\mathrm{H}$, we are now able to accurately determine the CEP of our laser pulses.

We perform quantitative analysis on the laser CEP effects by 'binning' both data and theoretical predictions to a width of $5 \mathrm{eV}$ in a compromise to improve the signalto-noise ratio at an acceptable expense to the energy resolution. These binned energy intervals, $B_{E}(\phi)$ demonstrate strong sinusoidal dependence of the laser CEP which we can fit to a function of the form:

$$
B_{E}(\phi)=A \sin \left(\phi+\phi_{0}\right),
$$

where $A$ is the amplitude of the waveform and $\phi_{0}$ is the phase 'offset' which is typically 
used to calibrate the CEP in phase-tagged experiments. The values of $\phi_{0}$ obtained from both the data and theory predictions are plotted in figure 16 at laser intensities of 1.2 and $2.5 \times 10^{14} \mathrm{~W} / \mathrm{cm}^{2}$ for atomic $\mathrm{H}$ and the noble gases.

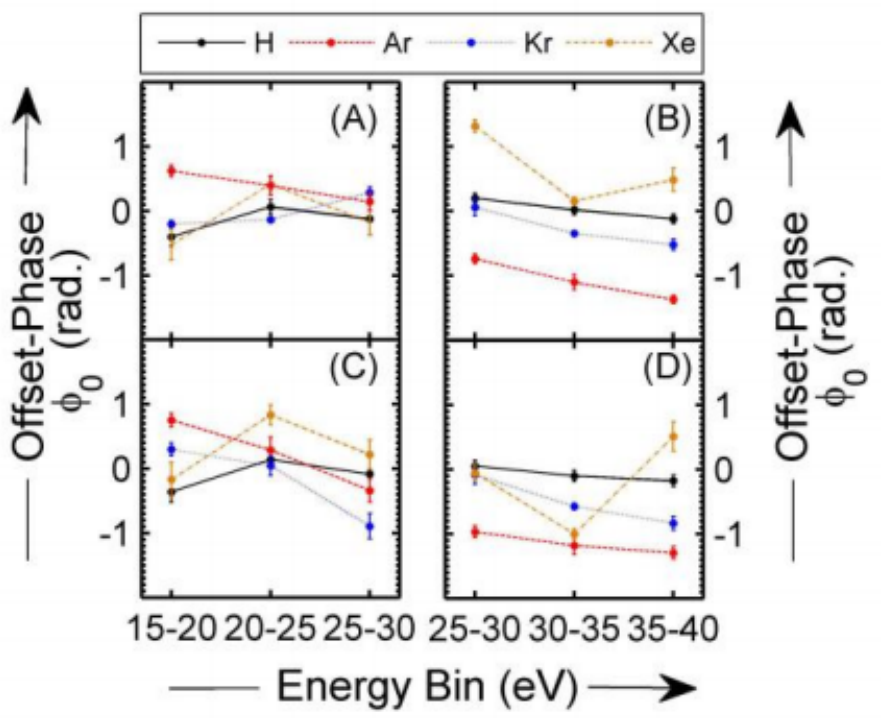

Figure 16: Phase offset $\phi_{0}$ values for $5 \mathrm{eV}$ binned experimental data (top row) and theoretical predictions (bottom row) at calibrated peak laser intensities of 1.2 (left column) and 2.5 (right column) in units of $\times 10^{14} \mathrm{~W} / \mathrm{cm}^{2}$. The $y$-axis is in units of rad. and dotted lines are used to guide the eye. Copyright (2017) by the American Physical Society [10].

As expected, the atomic $\mathrm{H} \phi_{0}$ values are in quantitative agreement between experiment and theory at both laser peak intensities, and to a lesser extent so too are the $\phi_{0}$ values of Ar. However, both $\mathrm{Kr}$ and Xe are markedly different from predictions based on 3D-TDSE under SAE, providing conclusive proof that there are systematic errors in the phase-tagged measurements using these gases as a means of laser CEP retrieval. The reference data presented here eliminates this systematic offset as it references accurate CEP measurements in the noble-gases back to certifiably accurate measurements in atomic $\mathrm{H}$.

\section{Summary and outlook}

The experiments on atomic $\mathrm{H}$ reviewed here have provided important benchmark measurements for strong-field ionization studies in the few-cycle regime. Current stateof-the-art measurements demonstrate the ability to resolve differences between common theoretical approximations used to model SFI. We have utilized atomic $\mathrm{H}$ as a target species in laser-matter interactions to precisely calibrate few-cycle pulses, enabling experimental groups with few-cycle lasers to accurately determine both the peak laser 
intensity and the carrier-envelope phase of their laser pulses. Using the reference data presented in [9] and [10] and reviewed herein, we hope to aid important investigations in SFI studies, such as the extremely topical and contested idea of the quantum tunneling time.

Acknowledgements Data collection was performed by the $\mathrm{H}$ experimental group at the AASF: William Wallace, Omair Ghafur, Champak Khurmi, Satya Sainadh U, James Calvert, and Dane Laban. We thank Professor Daniel Kleppner of the Massachusetts Institute of Technology for assistance in setting up the hydrogen source. The theoretical simulations were provided by a number of groups of collaborators: Professor Klaus Bartschat at Drake University working with Professor Alexei Grum-Grzhimailo of Lomonosov Moscow State University, Daniel Wells and Professor Harry Quiney of the University of Melbourne, Professor Xiao-Min Tong of the University of Tsukuba, and Igor Ivanov and Professor Anatoli Kheifets of the Australian National University. This work was supported by the U.S. Air Force Office of Scientific Research (FA2386-12-14025) and the Australian Research Council (ARC) Centre of Excellence for Coherent X-Ray Science (CEO561787) and Discovery Projects (DP110101894).

\section{References}

[1] P. Corkum and F. Krausz, Attosecond science, Nature Physics 3, 381 (2007).

[2] T. Popmintchev, M.-C. Chen, P. Arpin, M. M. Murnane and H. C. Kapteyn, The attosecond nonlinear optics of bright coherent x-ray generation, Nature Photonics 4, 822 (2010).

[3] L. Bergé, S. Skupin, R. Nuter, J. Kasparian and J.-P. Wolf, Ultrashort filaments of light in weakly ionized, optically transparent media, Reports on progress in physics 70, 1633 (2007).

[4] P. R. Hemmer, R. B. Miles, P. Polynkin, T. Siebert, A. V. Sokolov, P. Sprangle and M. O. Scully, Standoff spectroscopy via remote generation of a backward-propagating laser beam, Proceedings of the National Academy of Sciences 108, 3130 (2011).

[5] M. G. Pullen, W. C. Wallace, D. E. Laban, A. J. Palmer, G. F. Hanne, A. N. Grum-Grzhimailo, B. Abeln, K. Bartschat, D. Weflen, I. Ivanov, A. Kheifets, H. M. Quiney, I. V. Litvinyuk, R. T. Sang and D. Kielpinski, Experimental ionization of atomic hydrogen with few-cycle pulses, Optics letters 36, 3660 (2011).

[6] W. C. Wallace, M. G. Pullen, D. E. Laban, O. Ghafur, H. Xu, A. J. Palmer, G. F. Hanne, K. Bartschat, A. N. Grum-Grzhimailo, H. M. Quiney, I. V. Litvinyuk, R. T. Sang and D. Kielpinski, Carrier-envelope phase effects in above-threshold ionization of atomic hydrogen, New Journal of Physics 15, 033002 (2013).

[7] M. G. Pullen, W. C. Wallace, D. E. Laban, A. J. Palmer, G. F. Hanne, A. N. Grum-Grzhimailo, K. Bartschat, I. Ivanov, A. Kheifets, D. Wells, H. M. Quiney, X. M. Tong, I. V. Litvinyuk, R. T. Sang and D. Kielpinski, Measurement of laser intensities approaching $10^{15} \mathrm{~W} / \mathrm{cm}^{2}$ with an accuracy of 1\%, Phys. Rev. A 87, 053411 (2013).

[8] D. Kielpinski, R. Sang and I. Litvinyuk, Benchmarking strong-field ionization with atomic hydrogen, Journal of Physics B: Atomic, Molecular and Optical Physics 47, 204003 (2014).

[9] W. C. Wallace, O. Ghafur, C. Khurmi, S. Sainadh U, J. E. Calvert, D. E. Laban, M. G. Pullen, K. Bartschat, A. N. Grum-Grzhimailo, D. Wells, H. M. Quiney, X. M. Tong, I. V. Litvinyuk, R. T. Sang and D. Kielpinski, Precise and accurate measurements of strong-field photoionization and a transferable laser intensity calibration standard, Phys. Rev. Lett. 117, 053001 (2016).

[10] C. Khurmi, W. C. Wallace, S. Sainadh U, I. A. Ivanov, A. S. Kheifets, X. M. Tong, I. V. Litvinyuk, R. T. Sang and D. Kielpinski, Measuring laser carrier-envelope-phase effects in the noble gases with an atomic hydrogen calibration standard, Phys. Rev. A 96, 013404 (2017).

[11] I. BIPM, I. IFCC, I. IUPAC and O. ISO, The international vocabulary of metrology - basic and general concepts and associated terms (vim), 3rd edn. jcgm 200: 2012, JCGM (Joint Committee 
for Guides in Metrology) (2008).

[12] I. BIPM, I. IFCC, I. IUPAC and O. ISO, Evaluation of measurement data-guide for the expression of uncertainty in measurement. JCGM 100: 2008 (2008).

[13] T. Brabec and F. Krausz, Intense few-cycle laser fields: Frontiers of nonlinear optics, Reviews of Modern Physics 72, 545 (2000).

[14] T. Brabec and F. Krausz, Nonlinear optical pulse propagation in the single-cycle regime, Physical Review Letters 78, 3282 (1997).

[15] G. G. Paulus, F. Grasbon, H. Walther, P. Villoresi, M. Nisoli, S. Stagira, E. Priori and S. De Silvestri, Absolute-phase phenomena in photoionization with few-cycle laser pulses, Nature 414, 182 (2001).

[16] C. I. Blaga, J. Xu, A. D. DiChiara, E. Sistrunk, K. Zhang, P. Agostini, T. A. Miller, L. F. DiMauro and C. Lin, Imaging ultrafast molecular dynamics with laser-induced electron diffraction, Nature 483, 194 (2012).

[17] J. Wu, M. Meckel, L. P. H. Schmidt, M. Kunitski, S. Voss, H. Sann, H. Kim, T. Jahnke, A. Czasch and R. Dörner, Probing the tunnelling site of electrons in strong field enhanced ionization of molecules, Nature Communications 3, 1113 (2012).

[18] F. Grasbon, G. G. Paulus, H. Walther, P. Villoresi, G. Sansone, S. Stagira, M. Nisoli and S. De Silvestri, Above-threshold ionization at the few-cycle limit, Phys. Rev. Lett. 91, 173003 (2003).

[19] C. I. Blaga, F. Catoire, P. Colosimo, G. G. Paulus, H. G. Muller, P. Agostini and L. F. DiMauro, Strong-field photoionization revisited, Nature Physics 5, 335 (2009).

[20] J. Wassaf, V. Véniard, R. Taïeb and A. Maquet, Strong field atomic ionization origin of high-energy structures in photoelectron spectra, Phys. Rev. Lett. 90, 013003 (2003).

[21] P. Eckle, A. Pfeiffer, C. Cirelli, A. Staudte, R. Dörner, H. Muller, M. Büttiker and U. Keller, Attosecond ionization and tunneling delay time measurements in helium, science 322, 1525 (2008).

[22] L. Torlina, F. Morales, J. Kaushal, I. Ivanov, A. Kheifets, A. Zielinski, A. Scrinzi, H. G. Muller, S. Sukiasyan, M. Ivanov et al., Interpreting attoclock measurements of tunnelling times, Nature Physics 11, 503 (2015).

[23] W. C. Wallace, Strong-field ionisation of atomic hydrogen, Ph.D. thesis, Griffith University (2017).

[24] I. V. Litvinyuk, K. F. Lee, P. W. Dooley, D. M. Rayner, D. M. Villeneuve and P. B. Corkum, Alignment-dependent strong field ionization of molecules, Phys. Rev. Lett. 90, 233003 (2003).

[25] S. Micheau, Z. Chen, A. T. Le, J. Rauschenberger, M. F. Kling and C. D. Lin, Accurate retrieval of target structures and laser parameters of few-cycle pulses from photoelectron momentum spectra, Phys. Rev. Lett. 102, 073001 (2009).

[26] Z. Chen, T. Wittmann, B. Horvath and C. D. Lin, Complete real-time temporal waveform characterization of single-shot few-cycle laser pulses, Phys. Rev. A 80, 061402 (2009).

[27] Z. Chen, A.-T. Le, T. Morishita and C. D. Lin, Quantitative rescattering theory for laser-induced high-energy plateau photoelectron spectra, Phys. Rev. A 79, 033409 (2009).

[28] T. Udem, R. Holzwarth and T. W. Hänsch, Optical frequency metrology, Nature 416, 233 (2002).

[29] R. H. Neynaber, L. L. Marino, E. W. Rothe and S. M. Trujillo, Scattering of low-energy electrons by atomic hydrogen, Physical Review 124, 135 (1961).

[30] E. C. Samano, W. E. Carr, M. Seidl and B. S. Lee, An arc discharge hydrogen atom source, Review of scientific instruments 64, 2746 (1993).

[31] H. Koschmieder and V. Raible, Intense atomic-hydrogen beam source, Review of Scientific Instruments 46, 536 (1975).

[32] J. Slevin and W. Stirling, Radio frequency atomic hydrogen beam source, Review of Scientific Instruments 52, 1780 (1981).

[33] J. P. Schwonek, A study of a cold atomic hydrogen beam source, Ph.D. thesis, Massachusetts Institute of Technology (1990).

[34] B. P. Lavrov, A. V. Pipa and J. Röpcke, On determination of the degree of dissociation of hydrogen 
in non-equilibrium plasmas by means of emission spectroscopy: I. The collision-radiative model and numerical experiments, Plasma Sources Science and Technology 15, 135 (2006), ISSN 09630252.

[35] B. P. Lavrov, N. Lang, A. V. Pipa and J. Röpcke, On determination of the degree of dissociation of hydrogen in non-equilibrium plasmas by means of emission spectroscopy: II. Experimental verification, Plasma Sources Science and Technology 15, 147 (2006), ISSN 0963-0252.

[36] M. G. Pullen, Above threshold ionisation of atomic hydrogen using few-cycle pulses, Ph.D. thesis, Griffith University (2011).

[37] A. Stingl, R. Szipöcs, M. Lenzner, C. Spielmann and F. Krausz, Sub-10-fs mirror-dispersioncontrolled Ti:sapphire laser, Opt. Lett. 20, 602 (1995).

[38] P. Maine, D. Strickland, P. Bado, M. Pessot and G. Mourou, Generation of ultrahigh peak power pulses by chirped pulse amplification, IEEE Journal of Quantum Electronics 24, 398 (1988).

[39] R. L. Fork, C. H. B. Cruz, P. C. Becker and C. V. Shank, Compression of optical pulses to six femtoseconds by using cubic phase compensation, Opt. Lett. 12, 483 (1987).

[40] S. Sartania, Z. Cheng, M. Lenzner, G. Tempea, C. Spielmann, F. Krausz and K. Ferencz, Generation of 0.1-TW 5-fs optical pulses at a 1-kHz repetition rate, Opt. Lett. 22, 1562 (1997).

[41] T. Fuji, J. Rauschenberger, A. Apolonski, V. S. Yakovlev, G. Tempea, T. Udem, C. Gohle, T. W. Hänsch, W. Lehnert, M. Scherer and F. Krausz, Monolithic carrier-envelope phase-stabilization scheme, Opt. Lett. 30, 332 (2005).

[42] A. Apolonski, A. Poppe, G. Tempea, C. Spielmann, T. Udem, R. Holzwarth, T. W. Hänsch and F. Krausz, Controlling the phase evolution of few-cycle light pulses, Physical Review Letters 85, $740(2000)$.

[43] L. Xu, T. Hänsch, C. Spielmann, A. Poppe, T. Brabec and F. Krausz, Route to phase control of ultrashort light pulses, Optics letters 21, 2008 (1996).

[44] J.-C. M. Diels, J. J. Fontaine, I. C. McMichael and F. Simoni, Control and measurement of ultrashort pulse shapes (in amplitude and phase) with femtosecond accuracy, Appl. Opt. 24, 1270 (1985).

[45] W. E. Stephens, A pulsed mass spectrometer with time dispersion, Phys. Rev. 69, 691 (1946).

[46] A. E. Cameron and D. F. Eggers, An ion velocitron, Review of Scientific Instruments 19, 605 (1948).

[47] M. M. Wolff and W. E. Stephens, A pulsed mass spectrometer with time dispersion, Review of Scientific Instruments 24, 616 (1953).

[48] D. A. Howe, D. W. Allan and J. Barnes, Properties of signal sources and measurement methods, in Thirty Fifth Annual Frequency Control Symposium, pp. 669-716 (IEEE, 1981).

[49] M. V. Ammosov, N. B. Delone and V. Krainov, Tunnel ionization of complex atoms and of atomic ions in an alternating electromagnetic field, Soviet Physics JETP 64, 1191 (1986).

[50] X. M. Tong and C. D. Lin, Empirical formula for static field ionization rates of atoms and molecules by lasers in the barrier-suppression regime, Journal of Physics B: Atomic, Molecular and Optical Physics 38, 2593 (2005).

[51] X. M. Tong, K. Hino and N. Toshima, Phase-dependent atomic ionization in few-cycle intense laser fields, Phys. Rev. A 74, 031405 (2006).

[52] G. Paulus, F. Lindner, D. Milošević and W. Becker, Phase-controlled single-cycle strong-field photoionization, Physica Scripta 2004, 120 (2004).

[53] A. Sayler, M. Arbeiter, S. Fasold, D. Adolph, M. Möller, D. Hoff, T. Rathje, B. Fetić, D. Milošević, T. Fennel et al., Accurate determination of absolute carrier-envelope phase dependence using photo-ionization, Optics Letters 40, 3137 (2015).

[54] G. G. Paulus, F. Lindner, H. Walther, A. Baltuška, E. Goulielmakis, M. Lezius and F. Krausz, Measurement of the phase of few-cycle laser pulses, Physical review letters 91, 253004 (2003). 